\title{
THE CORANK AND THE INDEX ARE BLOW-NASH INVARIANTS
}

\author{
GOULWEN FichOU
}

\begin{abstract}
Is the corank an invariant of the blow-analytic equivalence between real analytic function germs? We give a positive answer in the case of the blow-Nash equivalence, which is a natural variant of the blow-analytic equivalence for Nash function germs. In addition, we prove that the index is also a blow-Nash invariant. Their proof is based on the computation of some virtual Poincaré polynomials and zeta functions associated to a Nash function germ.
\end{abstract}

\section{Introduction}

The classification of real analytic function germs is a difficult topic, notably in the choice of a good equivalence relation, between germs, to study. An interesting relation, called blow-analytic equivalence, has been introduced by $\mathrm{T}$. C. Kuo [10] and studied by several authors (see [5] for a survey). Notably, it has been proved that such an equivalence relation does not admit moduli for a family with isolated singularities. Moreover, the proof of this result produces effective methods to give blow-analytic triviality criteria. On the other hand, some invariants have been introduced in order to distinguish blow-analytic types (cf. [9]). However, it remains difficult to obtain effective classification results, at least in dimension greater than 3 , because of the complexity of these invariants.

In this paper, we address the following related question, raised by T. Fukui.

Let $f:\left(\mathbf{R}^{d}, 0\right) \rightarrow(\mathbf{R}, 0)$ be an analytic germ. Assume that the Jacobian matrix of $f$ at 0 does vanish. Let $r$ denote the rank of the Hessian of $f$ at 0 . Then $f$ is analytically equivalent to a function germ of the form

$$
\sum_{i=1}^{s} x_{i}^{2}-\sum_{j=s+1}^{s+t} x_{j}^{2}+F(x),
$$

where $s+t=r$ (note that $s$ or $t$ may vanish) and $F$ is an analytic germ with order at least 3. The corank of $f$ is defined to be the corank of its Hessian matrix at 0 , that is $d-r$.

1991 Mathematics Subject Classification. 14B05, 14P20, 14P25, 32 S15.

Received May 9, 2005; revised July 11, 2005. 
QUESTION. Is the corank of an analytic function germ an invariant of the blow-analytic equivalence?

The answer to such a question would be a step toward a better understanding of the blow-analytic equivalence relation, and therefore a better understanding of the singularities of real analytic function germs.

We will not give a complete answer to this question, but we answer a natural variant of the question for Nash function germs (i.e., germs that are analytic and semi-algebraic). One can define the blow-Nash equivalence between Nash function germs (see [4]; see also [6, 7, 8] for the case of Nash sets), and this relation still has good triviality properties and effective invariants called zeta functions. These zeta functions are defined, following the idea of motivic integration [3], using an additive and multiplicative invariant, under Nash isomorphisms, of real algebraic sets: the virtual Poincaré polynomial (cf. part 1.1).

The main result of this paper states that blow-Nash equivalent Nash function germs have the same corank (theorem 1.5). We prove moreover that their index coincide (the index is defined to be the integer $t$ above, which counts the number of minus signs in the quadratic part).

The proof is based on the invariance of the zeta functions with respect to the blow-Nash equivalence [4], and on the computation of a significant part of these zeta functions for germs of Nash functions of the type $\sum_{i=1}^{s} x_{i}^{2}-\sum_{j=s+1}^{s+t} x_{j}^{2}$ (cf part 3). To reach this aim, the computation of some virtual Poincaré polynomials associated to these germs is crucial. Such a computation may be difficult in general, but here we manage to conclude thanks to the degeneracy of a Leray-Serre spectral sequence (cf part 2).

Acknowledgments. The author does wish to thank Toshizumi Fukui for stimulating discussions during the invited stay of the author at Saitama University. He thanks also Michel Coste, Satoshi Koike and Adam Parusiński for valuable remarks.

\section{Corank and Blow-Nash equivalence}

1.1. Blow-Nash equivalence. In this section, we recall briefly the notion of blow-Nash equivalence as well as that of zeta functions. For more details, the reader is refer to [4].

DEFINITION 1.1.

(1) An algebraic modification of a Nash function germ $f:\left(\mathbf{R}^{d}, 0\right) \rightarrow(\mathbf{R}, 0)$ is a proper birational algebraic morphism $\sigma_{f}:\left(M_{f}, \sigma_{f}^{-1}(0)\right) \rightarrow\left(\mathbf{R}^{d}, 0\right)$, between semi-algebraic neighbourhoods of 0 in $\mathbf{R}^{d}$ and $\sigma_{f}^{-1}(0)$ in $M_{f}$, which is an isomorphism over the complement of the zero locus of $f$, and for which $f \circ \sigma$ is in normal crossing. 
(2) Let $f, g:\left(\mathbf{R}^{d}, 0\right) \rightarrow(\mathbf{R}, 0)$ be Nash function germs. They are said to be blow-Nash equivalent if there exist two algebraic modifications

$$
\sigma_{f}:\left(M_{f}, \sigma_{f}^{-1}(0)\right) \rightarrow\left(\mathbf{R}^{d}, 0\right) \text { and } \sigma_{g}:\left(M_{g}, \sigma_{g}^{-1}(0)\right) \rightarrow\left(\mathbf{R}^{d}, 0\right),
$$

such that $f \circ \sigma_{f}$ and jac $\sigma_{f}$ (respectively $g \circ \sigma_{g}$ and jac $\sigma_{g}$ ) have only normal crossings simultaneously, and a Nash isomorphism (i.e. a semialgebraic map which is an analytic isomorphism) $\Phi$ between semialgebraic neighbourhoods $\left(M_{f}, \sigma_{f}^{-1}(0)\right)$ and $\left(M_{g}, \sigma_{g}^{-1}(0)\right)$ which preserves the multiplicities of the Jacobian determinants of $\sigma_{f}$ and $\sigma_{g}$ along the components of the exceptional divisors, and which induces a homeomorphism $\phi:\left(\mathbf{R}^{d}, 0\right) \rightarrow\left(\mathbf{R}^{d}, 0\right)$ such that $f=g \circ \phi$, as illustrated by the commutative diagram:



The principal results, concerning this equivalence relation between Nash function germs, are the following. On one hand, it does not admit moduli for a Nash family with isolated singularities. On the other hand, we know invariants for the blow-Nash equivalence, called zeta functions. These invariants will be crucial in the proof of the main result of this paper. We recall now the definition of these zeta functions. To begin with, let us introduce the invariant of constructible real algebraic sets we will deal with.

By an additive map on the category of constructible real algebraic sets, we mean a map $\beta$ such that $\beta(X)=\beta(Y)+\beta(X \backslash Y)$ where $Y$ is an algebraic subset closed in $X$. Moreover $\beta$ is called multiplicative if $\beta\left(X_{1} \times X_{2}\right)=\beta\left(X_{1}\right) \cdot \beta\left(X_{2}\right)$ for constructible real algebraic sets $X_{1}, X_{2}$.

Proposition $1.2([4])$. For an integer $i$, there exists an additive map $\beta_{i}$ with values in $\mathbf{Z}$, defined on the category of constructible real algebraic sets. It coincides with the classical Betti number $\operatorname{dim} H_{i}\left(\cdot, \frac{\mathbf{Z}}{\mathbf{2} \mathbf{Z}}\right)$ on the connected component of compact nonsingular real algebraic varieties.

Moreover $\beta(\cdot)=\sum_{i \geq 0} \beta_{i}(\cdot) u^{i}$ is multiplicative, with values in $\mathbf{Z}[u]$. $\beta\left(X_{2}\right)$.

Finally, if $X_{1}$ and $X_{2}$ are Nash isomorphic real algebraic sets, then $\beta\left(X_{1}\right)=$

The invariant $\beta_{i}$ is called the $i$-th virtual Betti number, and the polynomial $\beta$ the virtual Poincaré polynomial. 
Then, using our virtual Poincaré polynomial, we can define the zeta functions of a Nash function germ $f:\left(\mathbf{R}^{d}, 0\right) \rightarrow(\mathbf{R}, 0)$ as follows. Denote by $\mathscr{L}$ the space of arcs at the origin $0 \in \mathbf{R}^{d}$, that is:

$$
\mathscr{L}=\mathscr{L}\left(\mathbf{R}^{d}, 0\right)=\left\{\gamma:(\mathbf{R}, 0) \rightarrow\left(\mathbf{R}^{d}, 0\right): \gamma \text { formal }\right\},
$$

and by $\mathscr{L}_{n}$ the space of arcs truncated at order $n+1$ :

$$
\mathscr{L}_{n}=\mathscr{L}_{n}\left(\mathbf{R}^{d}, 0\right)=\left\{\gamma \in \mathscr{L}: \gamma(t)=a_{1} t+a_{2} t^{2}+\cdots+a_{n} t^{n}, a_{i} \in \mathbf{R}^{d}\right\},
$$

for $n \geq 0$ an integer.

We define the naive zeta function $Z_{f}(T)$ of $f$ as the following element of $\mathbf{Z}\left[u, u^{-1}\right][[T]]:$

$$
Z_{f}(T)=\sum_{n \geq 1} \beta\left(A_{n}\right) u^{-n d} T^{n}
$$

where

$$
A_{n}=\left\{\gamma \in \mathscr{L}_{n}: \operatorname{ord}(f \circ \gamma)=n\right\}=\left\{\gamma \in \mathscr{L}_{n}: f \circ \gamma(t)=b t^{n}+\cdots, b \neq 0\right\} .
$$

Similarly, we define zeta functions with sign by

$$
Z_{f}^{+1}(T)=\sum_{n \geq 1} \beta\left(A_{n}^{+1}\right) u^{-n d} T^{n} \quad \text { and } \quad Z_{f}^{-1}(T)=\sum_{n \geq 1} \beta\left(A_{n}^{-1}\right) u^{-n d} T^{n},
$$

where

$$
\begin{gathered}
A_{n}^{+1}=\left\{\gamma \in \mathscr{L}_{n}: f \circ \gamma(t)=+t^{n}+\cdots\right\} \quad \text { and } \\
A_{n}^{-1}=\left\{\gamma \in \mathscr{L}_{n}: f \circ \gamma(t)=-t^{n}+\cdots\right\} .
\end{gathered}
$$

The crucial result concerning these zeta functions is the following:

THEOREM 1.3 ([4]). Blow-Nash equivalent Nash function germs have the same naive zeta function and the same zeta functions with sign.

1.2. Corank of a Nash function germ. The corank and the index of a Nash function germ is defined in the same way as in the smooth case. Moreover, the same splitting lemma holds in the Nash case.

Lemma 1.4. Let $f:\left(\mathbf{R}^{d}, 0\right) \rightarrow(\mathbf{R}, 0)$ be a Nash function germ. Assume that the Jacobian matrix of $f$ does vanish at 0 . Then there exist a Nash isomorphism $\phi:\left(\mathbf{R}^{d}, 0\right) \rightarrow\left(\mathbf{R}^{d}, 0\right)$ and integers $s, t$ (possibly equal to zero), where $s+t$ equals the rank of the Hessian matrix of $f$ at 0 , such that

$$
f \circ \phi\left(x_{1}, \ldots, x_{d}\right)=\sum_{i=1}^{s} x_{i}^{2}-\sum_{j=s+1}^{s+t} x_{j}^{2}+F\left(x_{s+t+1}, \ldots, x_{d}\right),
$$

where $F$ is a Nash function germ with order at least 3. 
Classical proofs of lemma 1.4, in the smooth case, use a parametrized version of the Morse Lemma [1]. But this is not allowed in the Nash setting since it requires integration along vector fields. However, an elementary proof, using only the implicit function theorem and the Hadamard division lemma, has been given in [11]. This method adapts to our case because the implicit function theorem does hold in the Nash setting [2], whereas the Hadamard division lemma is no longer necessary since we are working with analytic functions.

Let us state now the central result of this paper.

TheOREM 1.5. Let $f, g:\left(\mathbf{R}^{d}, 0\right) \rightarrow(\mathbf{R}, 0)$ be blow-Nash equivalent Nash function germs. Then $f$ and $g$ have the same corank and the same index.

Proof. The proof of theorem 1.5 consists in comparing the coefficient of $T^{2}$ of the zeta functions associated to $f$ and $g$. Due to the invariance theorem 1.3, it is sufficient to compare these coefficients for the simpler Nash function germs given by lemma 1.4, since Nash equivalent germs are automatically blow-Nash equivalent.

Now, the result follows from proposition 3.1 below, where we compute the coefficient of $T^{2}$ for the zeta functions of the polynomial germs given by the quadratic part of these simpler Nash germs. Actually, the coefficient of $T^{2}$ of a quadratic polynomial $q$ is the same as that of $q+F$, where $F$ has order at least 3, by definition of the sets of $\operatorname{arcs} A_{2}$ and $A_{2}^{ \pm 1}$.

Remark 1.6. There exist similar zeta functions that are invariants for the blow-analytic equivalence between real analytic germs (cf. [9]). These zeta functions can be derived from the one presented in this paper by evaluating the indeterminacy $u$ at -1 . Unfortunately, they are not sufficient to obtain the invariance of the corank for blow-analytic equivalence by using the method presented here.

\section{Computation of some virtual Poincaré polynomials}

Let $X_{m, M}$ be the real algebraic subset of $\mathbf{R}^{m+M}$ defined by the equation

$$
\sum_{i=1}^{m} x_{i}^{2}-\sum_{j=1}^{M} y_{j}^{2}=0 .
$$

In this section, we compute the value of the virtual Poincare polynomial $\beta\left(X_{m, M}\right)$ in terms of $m$ and $M$. This computation is based on the degeneracy of a LeraySerre spectral sequence associated to the projectivisation of $X_{m, M}$.

Without lost of generality, one may assume that $m \leq M$.

Proposition 2.1. If $m \geq 1$, then $\beta\left(X_{m, M}\right)=u^{m+M-1}-u^{M-1}+u^{m}$. 
Remark 2.2.

(1) If $m=0, X_{0, M}$ is reduced to a single point, and therefore $\beta\left(X_{0, M}\right)=1$.

(2) Note that if $m=1$, then the computation of $\beta\left(X_{1, M}\right)$ is easy since $X_{1, M}$ is just a cone based on a sphere. Thus $\beta\left(X_{1, M}\right)=1+(u-1)\left(1+u^{M-1}\right)$ $=u^{M}-u^{M-1}+u$.

(3) In the particular case where $m=M=2$, the computation can also be done in a simple way. Indeed $X_{2,2}$ is isomorphic to the variety given by $X Y=U V$ in $\mathbf{R}^{4}$. Then, for $Y \neq 0$, it defines a family of lines $X=\frac{U}{Y} V$, whereas for $Y=0$ we simply get $U V=0$. Thus

$\beta\left(X_{2,2}\right)=\beta\left(\mathbf{R}^{*} \times \mathbf{R}^{2}\right)+\beta(\{(X, U, V) \mid U V=0\})=(u-1) u^{2}+u(2 u-1)$.

Another nice way to compute this Poincaré polynomial is by using the toric structure of $X_{2,2}$. Actually, one see that $X_{2,2}$ is the union of the orbits under the torus action, that is $X_{2,2}$ is a disjoint union of $\left(\mathbf{R}^{*}\right)^{3}$, one point, and four copies of $\left(\mathbf{R}^{*}\right)^{2}$ and $\left(\mathbf{R}^{*}\right)$. Therefore, by additivity of $\beta$ :

$$
\beta\left(X_{2,2}\right)=(u-1)^{3}+4(u-1)^{2}+4(u-1)+1=u^{3}+u^{2}-u .
$$

Unfortunately, it does not seem possible to generalize this method.

The proof of proposition 2.1 is based on a reduction to the projective case. As a preliminary step, we compute the virtual Poincare polynomial of the projective subset $Z_{m, M}$ of $\mathbf{P}^{m+M-1}(\mathbf{R})$ defined by the same equation as that of $X_{m, M}$.

LemmA 2.3. Take $M \geq 2$. Let $Z_{m, M}$ be defined by $\sum_{i=1}^{m} x_{i}^{2}-\sum_{j=1}^{M} y_{j}^{2}=0$ in $\mathbf{P}^{m+M-1}(\mathbf{R})$. Then

$$
\beta\left(Z_{m, M}\right)=\left(1+u^{M-1}\right)\left(1+u+\cdots+u^{m-1}\right) .
$$

Proof. To begin with, note that $Z_{m, M}$ is nonsingular as a real algebraic set. Therefore the virtual Betti numbers of $Z_{m, M}$ coincide with its classical Betti numbers (cf. proposition 1.2).

In order to compute these Betti numbers, consider the projection from $Z_{m, M}$ onto $\mathbf{P}^{m-1}(\mathbf{R})$ defined by

$$
\left[x_{1}: \cdots: x_{m}: y_{1}: \cdots: y_{M}\right] \mapsto\left[x_{1}: \cdots: x_{m}\right] .
$$

It is a well-defined fibration with fibre isomorphic to the unit sphere $S^{M-1}$ in $\mathbf{R}^{M}$. Working with coefficients in $\mathbf{Z}_{2}$, the cohomological Leray-Serre spectral sequence associated with this fibration converges to the cohomology of $Z_{m, M}$ :

$$
E_{2}^{p, q}=H^{p}\left(\mathbf{P}^{m-1}(\mathbf{R}), H^{q}\left(S^{M-1}, \mathbf{Z}_{2}\right)\right) \Rightarrow H^{p+q}\left(Z_{m, M}, \mathbf{Z}_{2}\right) .
$$


However $H^{q}\left(S^{M-1}, \mathbf{Z}_{2}\right)$ is isomorphic to $\mathbf{Z}_{2}$ if $q=0$ and $q=M-1$, and is zero otherwise. Therefore the nonzero terms of $E_{2}^{p, q}$, all equal to $\mathbf{Z}_{2}$, are localized in two lines (they are represented by a star in the figure below).

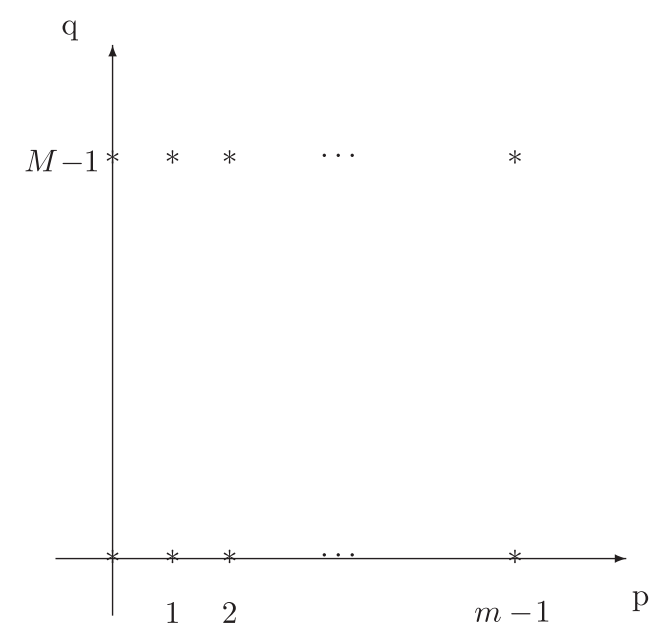

But, since $m \leq M$ by assumption, the spectral sequence degenerates and gives the Betti numbers of $Z_{m, M}$ (recall that $H_{i}\left(Z_{m, M}, \mathbf{Z}_{2}\right)$ is isomorphic to $\left.H^{i}\left(Z_{m, M}, \mathbf{Z}_{2}\right)\right)$. More precisely,

$$
\operatorname{dim} H_{i}\left(Z_{m, M}, \mathbf{Z}_{2}\right)= \begin{cases}1 & \text { if } i \in\{0, \ldots, m-1, M-1, \ldots, m+M-2\} \\ 0 & \text { otherwise }\end{cases}
$$

if $m<M$, and in the particular case where $m=M$, then

$$
\operatorname{dim} H_{i}\left(Z_{m, m}, \mathbf{Z}_{2}\right)= \begin{cases}1 & \text { if } i \in\{0, \ldots, m-2, m, \ldots, 2 m-2\} \\ 2 & \text { if } i=m-1 \\ 0 & \text { otherwise }\end{cases}
$$

So in general

$$
\begin{aligned}
\beta\left(Z_{m, M}\right) & =\left(1+u+\cdots+u^{m-1}\right)+\left(u^{M-1}+\cdots+u^{m+M-2}\right) \\
& =\left(1+u^{M-1}\right)\left(1+u+\cdots+u^{m-1}\right) .
\end{aligned}
$$

Remark 2.4. The result of lemma 2.3 can be stated as $\beta\left(Z_{m, M}\right)=$ $\beta\left(S^{M-1}\right) \beta\left(P^{m-1}(\mathbf{R})\right)$. This form suggests that the projection from $Z_{m, M}$ onto $P^{m-1}(\mathbf{R})$ could be a piecewise algebraically trivial fibration with fibre $S^{M-1}$, because of the additivity and multiplicativity of $\beta$. However it is not the case in general, as illustrated by the case $m=M=2$.

Let us explain now how we carry out the computation of the virtual Poincare polynomial of $X_{m, M}$ in terms of that of $Z_{m, M}$. 
Proof of proposition 2.1. It suffices to notice that the projection from $X_{m, M} \backslash\{0\}$ onto $Z_{m, M}$ is a piecewise algebraically trivial fibration with fibre $\mathbf{R}^{*}$. Therefore

$$
\beta\left(X_{m, M}\right)=1+(u-1) \beta\left(Z_{m, M}\right)
$$

by additivity and multiplicativity of the virtual Poincare polynomial $\beta$.

Now, note that $\beta\left(Z_{m, M}\right)=\left(1+u^{M-1}\right) \frac{u^{m}-1}{u-1}$, and so

$$
\beta\left(X_{m, M}\right)=1+\left(1+u^{M-1}\right)\left(u^{m}-1\right)=u^{m+M-1}-u^{M-1}+u^{m} .
$$

The following corollaries, which also specify the virtual Poincaré polynomial of some algebraic sets, will be useful for computing the zeta functions with sign in section 3 .

COROLlary 2.5. Let $X_{s, t}^{1}$ be the real algebraic subset of $\mathbf{R}^{s+t}$ defined by the equation

$$
\sum_{i=1}^{s} x_{i}^{2}-\sum_{j=1}^{t} y_{j}^{2}=1
$$

Assume that $s, t>0$.

- If $s \leq t$, then $\beta\left(X_{s, t}^{1}\right)=u^{t-1}\left(u^{s}-1\right)$.

- If $s>t$, then $\beta\left(X_{s, t}^{1}\right)=u^{t}\left(u^{s-1}+1\right)$.

Moreover $\beta\left(X_{0, t}^{1}\right)=0$ and $\beta\left(X_{s, 0}^{1}\right)=1+u^{s-1}$ if $s \geq 1$.

Proof. Let us begin with the case $s \leq t$. We homogenize the equation defining $X_{s, t}^{1}$. Then, we obtain a projective subset of $\mathbf{P}^{s+t}(\mathbf{R})$, denoted $Z_{s, t+1}$ in lemma 2.3, whose affine part is isomorphic to $X_{s, t}^{1}$, and whose part at infinity is isomorphic to $Z_{s, t}$. Therefore

$$
\begin{aligned}
\beta\left(X_{s, t}^{1}\right)=\beta\left(Z_{s, t+1}\right)-\beta\left(Z_{s, t}\right) & =\left(1+u^{t}\right)\left(1+\cdots+u^{s-1}\right)-\left(1+u^{t-1}\right)\left(1+\cdots+u^{s-1}\right) \\
& =u^{t-1}(u-1) \frac{u^{s}-1}{u-1}=u^{t-1}\left(u^{s}-1\right) .
\end{aligned}
$$

Now, let us turn to the case $s>t$. In the same way as above, by homogenisation of the equation defining $X_{s, t}^{1}$, we obtain a projective subset of $\mathbf{P}^{s+t}(\mathbf{R})$, denoted $Z_{t+1, s}$ (and not $Z_{s, t+1}$ because $s \geq t+1$ ), whose affine part is isomorphic to $X_{s, t}^{1}$. Moreover, the part at infinity is isomorphic to $Z_{t, s}$, therefore

$$
\beta\left(X_{s, t}^{1}\right)=\beta\left(Z_{t+1, s}\right)-\beta\left(Z_{t, s}\right),
$$

and the second member can be computed thanks to lemma 2.3. More precisely: 


$$
\begin{aligned}
\beta\left(X_{s, t}^{1}\right) & =\left(1+u^{s-1}\right)\left(1+u+\cdots+u^{t}\right)-\left(1+u^{s-1}\right)\left(1+u+\cdots+u^{t-1}\right) \\
& =u^{t}\left(1+u^{s-1}\right) .
\end{aligned}
$$

Finally, note that the set in consideration is empty in the case $s=0$, and isomorphic to a sphere in the case $t=0$.

We can rewrite corollary 2.5 as follows:

Corollary 2.6. Let $X_{s, t}^{-1}$ be the real algebraic subset of $\mathbf{R}^{s+t}$ defined by the equation

$$
\sum_{i=1}^{s} x_{i}^{2}-\sum_{j=1}^{t} y_{j}^{2}=-1
$$

Assume that $s, t>0$.

- If $s \geq t$, then $\beta\left(X_{s, t}^{-1}\right)=u^{s-1}\left(u^{t}-1\right)$.

- If $s<t$, then $\beta\left(X_{s, t}^{-1}\right)=u^{s}\left(u^{t-1}+1\right)$.

Moreover $\beta\left(X_{s, 0}^{-1}\right)=0$ and $\beta\left(X_{0, t}^{-1}\right)=1+u^{t-1}$ if $t \geq 1$.

\section{Proof of theorem 1.5}

We compute the coefficient of $T^{2}$ of the zeta functions with sign of a function germ $f:\left(\mathbf{R}^{d}, 0\right) \rightarrow(\mathbf{R}, 0)$ of the form

$$
f\left(x_{1}, \ldots, x_{s}, y_{1}, \ldots, y_{t}, z_{1}, \ldots, z_{d-s-t}\right)=\sum_{i=1}^{s} x_{i}^{2}-\sum_{j=1}^{t} y_{j}^{2} .
$$

Proposition 3.1 below states that the coefficients of $T^{2}$ of the zeta functions with sign determine the integers $s$ and $t$. It completes the proof of theorem 1.5.

Proposition 3.1. For a polynomial germ $f:\left(\mathbf{R}^{d}, 0\right) \rightarrow(\mathbf{R}, 0)$ of the form

$$
f\left(x_{1}, \ldots, x_{s}, y_{1}, \ldots, y_{t}, z_{1}, \ldots, z_{d-s-t}\right)=\sum_{i=1}^{s} x_{i}^{2}-\sum_{j=1}^{t} y_{j}^{2},
$$

the coefficients of $T^{2}$ of the zeta functions with sign of $f$ determine $s$ and $t$.

Proof. The space of truncated $\operatorname{arcs} A_{2}^{+1}(f)$ is isomorphic to the product

$$
\mathbf{R}^{2(d-s-t)} \times \mathbf{R}^{s+t} \times X_{s, t}^{1} .
$$

Actually, for an arc $\left(a_{1} t+b_{1} t^{2}, \ldots, a_{d} t+b_{d} t^{2}\right)$ in $A_{2}^{+1}(f)$, the first term of the product corresponds to the choice of the coefficients $a_{s+t+1}, b_{s+t+1}, \ldots, a_{d}, b_{d}$, the second to the choice of $b_{1}, \ldots, b_{s+t}$ and finally $X_{s, t}^{1}$ to the choice of $a_{1}, \ldots, a_{s+t}$. 
Due to corollary 2.5 , the quotient of $\beta\left(A_{2}^{+1}(f)\right)$ after dividing by the maximal power of $u$, is a polynomial of the form $u^{k}+1$ or $u^{k}-1$. Now, by corollary 2.5 again, it follows that $s=k+1$ in the former case, and $s=k$ in the latter one.

Similarly, $A_{2}^{-1}(f)$ is isomorphic to

$$
\mathbf{R}^{2(d-s-t)} \times \mathbf{R}^{s+t} \times X_{s, t}^{-1},
$$

and once more, after dividing $\beta\left(A_{2}^{-1}(f)\right)$ by the maximal power of $u$, we obtain a polynomial of the type $u^{l}+1$ or $u^{l}-1$. In the former case, then $t=l+1$ whereas in the latter one $t=l$.

Remark 3.2. The same method, applied to the coefficient of $T^{2}$ in the naive zeta function instead of in the zeta functions with sign, no longer gives such a determination. More precisely, what we still determine is $m=\min \{s, t\}$ and $M=\max \{s, t\}$, unless $m=0$ where we can not specify the value of $M$, or $M=m+1$ where we can not even specify the value of $m$ and $M$.

\section{REFERENCES}

[ 1 ] V. Arnold, S. Goussein-Zadé ANd A. Varchenko, Singularités des applications différentiables, Editions MIR, Moscou, 1986.

[2] J. BochnaK, M. Coste AND M.-F. Roy, Real algebraic geometry, Springer-Verlag, Berlin, 1998.

[ 3 ] J. Denef And F. Loeser, Germs of arcs on singular algebraic varieties and motivic integration, Invent. Math. 135 (1999), 201-232.

[4] G. FICHOU, Motivic invariants of arc-symmetric sets and blow-Nash equivalence, Compositio Math. 141 (2005), 655-688.

[5] T. FukuI, S. KoIke AND T.-C. Kuo, Blow-analytic equisingularities, properties, problems and progress, Real analytic and algebraic singularities (T. Fukuda, T. Fukui, S. Izumiya and S. Koike, eds.), Pitman research notes in mathematics series 381, 1998, 8-29.

[6] T. Fukui, S. Koike and M. Shiota, Modified Nash triviality of a family of zero-sets of real polynomial mappings, Ann. Inst. Fourier 48 (1998), 1395-1440.

[ 7 ] S. KolKe, Modified Nash triviality theorem for a family of zero-sets of weighted homogeneous polynomial mappings, J. Math. Soc. Japan 49 (1997), 617-631.

[8] S. Korke, Nash trivial simultaneous resolution for a family of zero-sets of Nash mappings, Math. Zeitschrift 234 (2000), 313-338.

[ 9 ] S. Koike and A. Parusiński, Motivic-type invariants of blow-analytic equivalence, Ann. Inst. Fourier 53 (2003), 2061-2104.

[10] T.-C. Kuo, On classification of real singularities, Invent. Math. 82 (1985), 257-262.

[11] S. López de Medrano, A splitting lemma for $C^{r}$ functions, $r \geq 2$, Singularity theory (Trieste, 1991), 444-450, World Sci. Publishing, River Edge, NJ, 1995.

InStitut MathÉmatiques De RenNeS

UNIVERSITÉ DE RENNES 1

CAMPUS DE BEAUlieu

35042 RENNES CEDEX

France

E-mail: goulwen.fichou@univ-rennes1.fr 